\title{
Geometric characteristics of semi-Fredholm operators and their asymptotic behaviour
}

by

JAROSLAV ZEMÁNEK (Warszawa)

Abstract. In this paper we obtain asymptotic formulas for the semi-Fredholm radius of a linear operator on a Banach space in terms of various essential minimum moduli of the perator. In particular, the results show that the classical stability theorem of T. Kato for semiFredholm operators is asymptotically sharp.

\section{Introduction}

Let $T$ be a bounded linear operator on an infinite-dimensional complex Banach space. We denote by $N(T)$ the null space and by $R(T)$ the range of $T$. The operator $T$ is called semi-Fredholm if either $\operatorname{dim} N(T)$ is finite and $R(T)$ is closed (a $\Phi_{+}$-operator), or codim $R(T)$ is finite (a $\Phi_{-}$-operator). We shall always look on operators as elements of $B(X)$, the Banach algebra of all bounded linear operators on $X$.

Since the set of semi-Fredholm operators is open in $B(X)$, we can define $d(T)$ to be the radius of the largest open ball centred at $T$ and contained in the semi-Fredholm operators (letting $d(T)=0$ if $T$ is not semi-Fredholm). This quantity was introduced in [15] and called the essential minimum modulus because of its spectral interpretation for Hilbert space operators. The semi-Fredholm radius $s(T)$ is the supremum of all $\varepsilon \geqslant 0$ such that the operators $T-\lambda I$ are semi-Fredholm for $|\lambda|<\varepsilon$. Clearly, we have

$$
d(T) \leqslant s(T) \text {. }
$$

Analogously we define the $\Phi_{+}$-radius $s_{+}(T)$ and the $\Phi_{-}$-radius $s_{-}(T)$ noting that $s(T)=\max \left\{s_{+}^{-}(T), s_{-}(T)\right\}$; if poth $s_{+}(T)$ and $s_{-}(T)$ are positive, then they are equal. First formulas for these radii were conjectured in [15] and proved in [16]. In this paper we continue this study.

AMS subject classification (1980): 47A55, 47A53. 
Our main concern here will be to show how the semi-Fredholm radius is related to the reduced minimum modulus

$$
\gamma(T)=\inf \{\|T x\|: \operatorname{dist}(x, N(T))=1\} .
$$

One of the classical results in the perturbation theory of semi-Fredholm operators is the following stability theorem due to T. Kato [4], Theorem 1: if $T$ is a seni-Fredholm operator and $S$ is arbitrary with $\|S\|<\gamma(T)$, then $T+S$ is also semi-Fredholm. In other words, Kato's theorem says that

$$
\gamma(T) \leqslant d(T)
$$

for every semi-Fredholm operator $T$.

It is well known that an operator $T$ is semi-Fredholm if and only if $T+F$ is semi-Fredholm for any finite rank operator $F$. It follows that $d(T+F)=d(T)$ for any such $F$. Letting

$$
\gamma_{\infty}(T)=\sup \{\gamma(T+F): \operatorname{rank} F<\infty\},
$$

(2) then implies

$$
\gamma_{\infty}(T) \leqslant d(T)
$$

We shall show that the Kato inequality (3) is asymptotically sharp in the sense that

$$
\lim _{n} \gamma_{\infty}\left(T^{n}\right)^{1 / n}=\lim _{n} d\left(T^{n}\right)^{1 / n}=s(T)
$$

for every semi-Fredholm operator $T$.

We shall also compare the reduced and essential minimum moduli to four other geometric characteristics of semi-Fredholm operators defined in Section 2. Two of these were studied by M. Schechter [11], without considering their asymptotic behaviour. It turns out that these characteristics are natural extensions of the so-called Gelfand, Kolmogorov, Bernstein and Mityagin numbers appearing in the literature even earlier; for a good survey of these numbers we refer to A. Pietsch [8]. After establishing appropriate geometric inequalities (Sections 5 and 6) and stability properties (Section 3) the proof of (4) as well as five other formulas are then obtained, in Section 8, by using two basic tools: the formula for the surjectivity or injectivity radius of a linear operator [6] combined with the method of removing jumping points in the semi-Fredholm domain (Section 7).

Finally we note that we have obtained the second equality in (4) in [16] by a different method, together with other related results' not covered here. The present approach, however, gives results which are even stronger than formulas (4), cf. Theorems 8.1 and 8.3 in this paper.

\section{The geometric characteristics}

Although we are interested exclusively in operators acting on one Banach space (because of studying the asymptotic behaviour of their iterates) we shall sometimes consider restrictions of an operator to certain subspaces, or compressions to certain quotient spaces. For an operator $T$ acting from a Banach space $X$ into a Banach space $Y$ we define the minimum modulus

$$
m(T)=\inf \{\|T x\|:\|x\|=1\},
$$

and the surjection modulus

$$
q(T)=\sup \left\{\varepsilon \geqslant 0: T U_{X} \supset \varepsilon U_{Y}\right\},
$$

where $U$ denotes the closed unit ball. For the adjoint operator $T^{\prime}$ acting from $Y^{\prime}$ to $X^{\prime}$ we recall the following useful relations:

$$
m\left(T^{\prime}\right)=q(T) \quad \text { and } \quad q\left(T^{\prime}\right)=m(T) ;
$$

see [9], Proposition B.3.8. Equivalent geometric interpretations of these characteristics can be found in [6]. We note that the reduced minimum modulus $\gamma(T)$ coincides with $m(T)$ or $q(T)$ if the operator $T$ is bounded from below or surjective, respectively.

We also adopt the following notation from [8]. If $W$ is a closed subspace of $X$ we denote by $J_{W}$ the embedding map of $W$ into $X$, and by $Q_{W}$ the canonical map of $X$ onto the quotient space $X / W$.

Let us recall the definitions of:

Gelfand numbers:

$$
c_{n}(T)=\inf \left\{\left\|T J_{W}\right\|: \operatorname{codim} W<n\right\},
$$

Kolmogorov numbers:

$$
k_{n}(T)=\inf \left\{\left\|Q_{V} T\right\|: \operatorname{dim} V<n\right\},
$$

Bernstein numbers

$$
u_{n}(T)=\sup \left\{m\left(T J_{W}\right): \operatorname{dim} W \geqslant n\right\},
$$

Mityagin numbers:

$$
v_{n}(T)=\sup \left\{q\left(Q_{V} T\right): \operatorname{codim} V \geqslant n\right\} .
$$

We note that everywhere $V$ and $W$ denote closed subspaces, and $n=1,2, \ldots$ Since these quantities are special cases of the $s$-numbers [8], we have by [5] and [14] the following formulas valid for every $T$ in $B(X)$ and each fixed $n=1,2, \ldots$ :

$$
\lim _{m} c_{n}\left(T^{m}\right)^{1 / m}=\lim _{m} k_{n}\left(T^{m}\right)^{1 / m}=\lim _{m} u_{n}\left(T^{m}\right)^{1 / m}=\lim _{m} v_{n}\left(T^{m}\right)^{1 / m}=\left|\lambda_{n}(T)\right|,
$$


where

$$
\left|\lambda_{1}(T)\right| \geqslant\left|\lambda_{2}(T)\right| \geqslant \ldots \geqslant|T|_{(t)}
$$

are (the absolute values of) the eigenvalues of $T$ greater than the essential spectral radius $|T|_{\omega}$, counted with their algebraic multiplicities; if there are only $n(=0,1,2, \ldots)$ such eigenvalues we formally define $\mid \lambda_{n+1}(T)$ $=\left|\lambda_{n+2}(T)\right|=\ldots=|T|_{\omega}$. We refer to [14] for details.

The four sequences defined above are clearly decreasing with respect to $n$, and are bounded from below by the following quantities, respectively:

$$
\begin{aligned}
& c(T)=\inf \left\{\left\|T J_{W}\right\|: \operatorname{codim} W<\infty\right\}, \\
& k(T)=\inf \left\{\left\|Q_{V} T\right\|: \operatorname{dim} V<\infty\right\}, \\
& u(T)=\sup \left\{m\left(T J_{W}\right): \operatorname{dim} W=\infty\right\}, \\
& v(T)=\sup \left\{q\left(Q_{V} T\right): \operatorname{codim} V=\infty\right\}
\end{aligned}
$$

We note that the functions $c$ and $k$ are measures of non-compactness, because we have $c(T)=0$ (or $k(T)=0$ ) if and only if $T$ is a compact operator; cf. [8], Theorem 9.3. Similarly, the function $u$ is a measure of nonstrict-singularity [9], 1.9.2, while the function $v$ is a measure of non-strictcosingularity [9], 1.10.2. Asymptotically these functions approach the essential spectral radius. The formulas

$$
\lim c\left(T^{m}\right)^{1 / m}=\lim k\left(T^{m}\right)^{1 / m}=|T|_{\omega}
$$

are due to L.S. Goldenstein, I. C. Gohberg and A. S. Markus (cf. [14]) The formula

$$
\lim u\left(T^{m}\right)^{1 / m}=|T|_{\omega}
$$

is given in [11], Theorem 2.17 , and the remaining one

$$
\lim _{m} v\left(T^{m}\right)^{1 / m}=|T|_{\omega}
$$

can be proved similarly.

We also note that $c(T)$ and $k(T)$ are exactly the greatest lower bounds of the corresponding sequences, while $u(T)$ and $v(T)$ are in general less than the corresponding infima. For if they were equal to them we would have $v(T)=u\left(T^{\prime}\right)$ by [8], Theorem 6.4 , and also $u(T)=v\left(T^{\prime}\right)$ by the remark in $[8]$, p. 213 , which can be proved using the strong version of the principle of local reflexivity:(K. D. Kürsten, references can be found in [9]). But these equalities cannot be true because if $T$ is strictly singular (consingular), then $T^{\prime}$ does not have to be strictly cosingular (singular); examples can be found in [7]. Having only the obvious inequalities $v\left(T^{\prime}\right) \geqslant u(T)$ and $u\left(T^{\prime}\right) \geqslant v(T)$ it is interesting to know that the asymptotic behaviour of the functions $u$ and $v$ is the same as described above.

The process started with the Gelfand, Kolmogorov, Bernstein and Mityagin numbers can naturally be continued in the following way. Let us define

$$
\begin{aligned}
G(T) & =\inf \left\{\left\|T J_{W}\right\|: \operatorname{dim} W=\infty\right\} \leqslant c(T), \\
K(T) & =\inf \left\{\left\|Q_{V} T\right\|: \operatorname{codim} V=\infty\right\} \leqslant k(T), \\
B(T) & =\sup \left\{m\left(T J_{W}\right): \operatorname{codim} W<\infty\right\} \leqslant u(T), \\
M(T) & =\sup \left\{q\left(Q_{V} T\right): \operatorname{dim} V<\infty\right\} \leqslant v(T) .
\end{aligned}
$$

Here again $V$ and $W$ denote closed subspaces while the letters $G, K, B$, and $M$ indicate that the corresponding quantity was derived from the Gelfand, Kolmogorov, Bernstein, and Mityagin numbers, respectively. As for the $s$-numbers (cf. [8], Theorem 8.1) we have

$$
G(T) \geqslant B(T) \quad \text { and } \quad K(T) \geqslant M(T) .
$$

The first inequality is given in [11], Theorem 2.19 , and the second one can be proved similarly (or, it immediately follows from $G\left(T^{\prime}\right) \geqslant B\left(T^{\prime}\right)$ by using Theorem 4.2 (ii) and (iv) of this paper).

The characteristics $G, B$, and $u$ were studied by $\mathbf{M}$. Schechter [11] (the notation used there is $\Gamma, v$, and $\tau$, respectively). Most of the arguments given there can be carried over to the characteristics $K, M$, and $v$. We only note that the function

$$
\Delta(T)=\sup _{M} \inf _{N \subset M}\left\|T J_{N}\right\|
$$

which plays an important role in Schechter's arguments and where $M, N$ are infinite-dimensional subspaces of $X$, is to be replaced by

$$
\nabla(T)=\sup _{W} \inf _{V \supset W}\left\|Q_{V} T\right\|
$$

where $V, W$ represent closed subspaces of infinite codimension in $X$. For the sake of completeness we note that

$$
\text { - }
$$

$$
\lim _{n} \Delta\left(T^{n}\right)^{1 / n}=\lim _{n} \nabla\left(T^{n}\right)^{1 / n}=|T|_{\omega}
$$

though we shall make no use of these formulas in this paper. Some of Schechter's results on $\Phi_{+}$-operators augmented by their $\Phi_{-}$-counterparts will be quoted when necessary. Obviously, we have $s(T) \leqslant|T|_{\omega}$. We are going to prove in Section 8 that

$$
\lim _{n} B\left(T^{n}\right)^{1 / n}=\lim _{n} G\left(T^{n}\right)^{1 / n}=s_{+}(T)
$$


and

$$
\lim _{n} M\left(T^{n}\right)^{1 / n}=\lim _{n} K\left(T^{n}\right)^{1 / n}=S_{\cdots}(T)
$$

for every $T$ in $B(X)$.

\section{Stability of the characteristics}

3.1. Theorem. Let $T$ be an arbitrary operator and $C$ be a compact operator. Then we have

(i) $G(T+C)=G(T)$,

(ii) $K(T+C)=K(T)$

(iii) $B(T+C)=B(T)$

(iv) $M(T+C)=M(T)$.

Proof. Property (i) follows from [11], Theorems 2.1 and 2.10, because the latter implies $\Delta(C)=0$. Property (ii) is a consequence of the corresponding dual results which can be proved similarly to those in [11] with $G$ replaced by $K, \Delta$ by $\nabla$, and $c$ by $k$.

Let us prove property (iv) which will be important in proving the formulas promised at the end of the preceding section. Given $:>0$ there exists, by definition of $M(T)$, a finite-dimensional subspace $V$ of $X$ such that

$$
M(T)-\varepsilon<q\left(Q_{V} T\right) \leqslant M(T) .
$$

Since the operator $C$ is compact, there exists a finite $\varepsilon$-net in $X$ for the set $C U$, the image of the unit ball. Let $W$ be a finite-dimensional subspace of $X$ containing this $\varepsilon$-net. Then we clearly have $\left\|Q_{W} C\right\| \leqslant \varepsilon$. Finally, we let $L$ $=V+W$. Then $\operatorname{dim} L$ is finite and we have

$$
\begin{aligned}
M(T)-\varepsilon & <q\left(Q_{V} T\right) \leqslant q\left(Q_{L} T\right) \leqslant q\left(Q_{L}(T+C)\right)+\left\|Q_{L} C\right\| \\
& \leqslant q\left(Q_{L}(T+C)\right)+\left\|Q_{W} C\right\| \leqslant q\left(Q_{L}(T+C)\right)+\varepsilon \leqslant M(T+C)+\varepsilon .
\end{aligned}
$$

Since $\varepsilon>0$ was arbitrary, we arrive at $M(T) \leqslant M(T+C)$. Replacing in this inequality the operator $T$ by $T+C$, and $C$ by $-C$, we get $M(T+C) \leqslant M(T)$. This proves (iv).

Property (iii) can be proved similarly by using a lemma of E. Lacey [2] p. 85 , but it also immediately follows from (iv) and Theorem 4.2 (iii) below.

\section{Quantitative characterizations of semi-Fredholm operators}

4.1. Theorem. For every operator $T$ the following three conditions are equivalent:

(i) $K(T)>0$,

(ii) $M(T)>0$,

(iii) $T$ is a $\Phi_{-}$-operator.

Analogous characterizations in terms of $G(T)$ and $B(T)$ hold for $\Phi_{+}-$operators.
Proof. Suppose that $T$ is a $\Phi$-operator. Then its range $R(T)$ is a closed subspace of finite codimension in $X$. Let $V$ be a finite-dimensional subspace in $X$ such that $X=V \oplus R(T)$. Then the operator $Q_{V} T$ is surjective, and hence $q\left(Q_{V} T\right)>0$. This shows that (iii) implies (ii). Next, (ii) implies (i) by the second inequality in (6).

It remains to show that (i) implies (iii). Suppose that $T$ is not a $\Phi$ - -operator. By a theorem of A. Lebow and M. Schechter (cf. [1], Theorem 4.4.10) there is a compact operator $C$ such that $V=R(T+C)^{-}$has infinite codimension in $X$. Since $Q_{V}(T+C)=0$ we conclude that $K(T+C)=0$. By Theorem 3.1 (ii) this implies $K(T)=0$.

The characterization of $\Phi_{+}$-operators can be proved similarly. It was given in [11] with a different argument.

The well-known duality between $\Phi_{+}$-operators and $\Phi_{-}$-operators ([1], p. 8) can be given the following quantitative form.

4.2. THEOREM. The following relations hold for every operator $T$ and its adjoint $T^{\prime}$ :

(i) $G(T) \geqslant K\left(T^{\prime}\right)$

(ii) $K(T) \geqslant G\left(T^{\prime}\right)$

(iii) $B(T)=M\left(T^{\prime}\right)$,

(iv) $M(T) \leqslant B\left(T^{\prime}\right)$

Proof. We choose a subspace $W$ (or $V$ ) appearing in the definition of a left-side characteristic, and denote by $V=W^{\perp}$ (or $W=V^{\perp}$ ) its annihilator in $X^{\prime}$. Considering the operators $T J_{W}$ and $Q_{V} T^{\prime}$ (or, $Q_{V} T$ and $T^{\prime} J_{W}$ ), we obtain equalities between their norms or minimum and surjection moduli (by (5)), respectively. Thus every term appearing in the definition of a left-side characteristic is equal to some one appearing in the definition on the right. This gives the corresponding inequalities between the characteristics. In the case (iii) the argument can be reversed because for a finite-dimensional subspace $V$ of $X^{\prime}$ we have $V=W^{\perp}$ with $W={ }^{\perp} V$ of finite codimension in $X$.

\section{Perturbing semi-Fredholm operators}

In this section we show that the four semi-Fredholm characteristics do not exceed the essential minimum modulus $d(T)$. By the way we give some more stability results. The first one completes a result of $M$. Schechter [11], Theorem 2.14 .

5.1. Theorem. Let $T, S$ be arbitrary elements of $B(X)$. If $v(S)<M(T)$, then $T+S$ is a $\Phi_{\text {-.. }}$-operator. If $u(S)<B(T)$, then $T+S$ is a $\Phi_{+}$-operator.

Proof. Suppose that $T+S$ is not a $\Phi_{\text {... }}$-operator. By the theorem of A. Lebow and $M$. Schechter [1], Theorem 4.4.10, there exists a compact operator $C$ such that the subspace $Y=R(T+S+C)^{-}$has infinite codimension in $X$ 
Let $\varepsilon>0$ be given. As in the proof of Theorem 3.1 (iv) we find a finitedimensional subspace $W$ in $X$ such that $\left\|Q_{W} C\right\| \leqslant \varepsilon$. Let $V$ be an arbitrary finite-dimensional subspace of $X$. Let $Z=Y+V+W$. Then $Z$ is a closed subspace of infinite codimension in $X$, and $Q_{Z}(T+S+C)=0$. Hence

$$
\begin{aligned}
q\left(Q_{V} T\right) & \leqslant q\left(Q_{Z} T\right)=q\left(Q_{Z}(S+C)\right) \leqslant q\left(Q_{Z} S\right)+\left\|Q_{Z} C\right\| \\
& \leqslant q\left(Q_{Z} S\right)+\left\|Q_{W} C\right\| \leqslant v(S)+\varepsilon,
\end{aligned}
$$

so by the definition of $M(T)$ we get $M(T) \leqslant v(S)+\varepsilon$. Since $\varepsilon>0$ was arbitrary, this gives a contradiction with the assumption $v(S)<M(T)$.

The second part was proved in [11], Theorem 2.14.

5.2. Theorem. Let $T, S$ be arbitrary elements of $B(X)$. If $c(S)<G(T)$, then $T+S$ is a $\Phi_{+}$-operator. If $k(S)<K(T)$, then $T+S$ is a $\Phi_{-}$-operator.

Proof. Suppose that $T+S$ is not a $\Phi_{+}$-operator. By a theorem of $\mathrm{M}$. Schechter [1], Theorem 4.4.7, there is a compact operator $C$ such that the nullspace $Y=N(T+S+C)$ is infinite-dimensional. Given $\varepsilon>0$, there is a closed subspace $W$ having finite codimension in $X$ such that $\left\|C J_{W}\right\| \leqslant \varepsilon$, cf. [2], p. 85. Let $V$ be any closed subspace of finite codimension in $X$, and set $Z=Y \cap V \cap W$. Then, $\operatorname{dim} Z$ is infinite and $(T+S+C) J_{Z}=0$, hence

$$
\left\|S J_{V}\right\| \geqslant\left\|S J_{Z}\right\| \geqslant\left\|T J_{Z}\right\|-\left\|C J_{Z}\right\| \geqslant G(T)-\varepsilon .
$$

So by the definition of $c(S)$ we conclude that $c(S) \geqslant G(T)-\varepsilon$. Since $\varepsilon>0$ was arbitrary, we get a contradiction.

The proof of the second part is similar to the preceding two arguments.

Remark. The assumptions of the preceding theorem can be replaced by weaker ones, for instance by $\Delta(S)<G(T)$ and $V(S)<K(T)$, respectively; cf. [11], Theorems 2.12 and 2.10. It is also enough to assume that the essential norm of $S$ is less than $G(T)$ or $K(T)$, respectively. Another stability result can be found in [16], Theorem 4.

5.3. Theorem. For every $T$ in $B(X)$ we have $\max \{G(T), K(T)\} \leqslant d(T)$.

Proof. If $T$ is not semi-Fredholm, then both the sides are zero. Suppose that $T$ is a $\Phi_{+}$-operator ( $\Phi_{-}$-operator). By Theorem 4.1 we have $G(T)>0(K(T)>0)$. Let $S$ be any operator with $\|S\|<G(T)(\|S\|<K(T))$. Since $c(S) \leqslant\|S\|(k(S) \leqslant\|S\|)$, we conclude by Theorem 5.2 that $T+S$ is a $\Phi_{+}$-operator ( $\Phi_{-}$-operator). Thus the open ball of radius $\max \{G(T), K(T)\}$ centred at $T$ is contained in the set of semi-Fredholm operators. This was to be proved.

\section{Geometric inequalities}

It is mentioned in [11] that B. Gramsch proved for any $\Phi_{+}$-operator $T$ the inequality $\gamma(T) \leqslant G(T)$. By virtue of the Kato property $\gamma(T)=\gamma\left(T^{\prime}\right)$ and Theorem 4.2 (ii) this yields $\gamma(T) \leqslant K(T)$ for any $\Phi_{\ldots .}$-operator $T$. By Theorem
3.1 we then obtain $\gamma_{\infty}(T) \leqslant G(T)$ or $\gamma_{\infty}(T) \leqslant K(T)$ provided that $T$ is a $\Phi_{+}-$ operator or a $\Phi_{-}$-operator, respectively. In particular, we have $\gamma_{\infty}(T) \leqslant \max \{G(T), K(T)\}$ for any semi-Fredholm operator $T$. We now give a lower estimate of $\gamma_{\infty}(T)$.

6.1. Theorem. For every $T$ in $B(X)$ we have $\gamma_{\infty}(T) \geqslant \max \{B(T), M(T)\}$.

Proof. If $T$ is not semi-Fredholm, then the assertion is true by Theorem 4.1. Supposing that $T$ is a $\Phi_{+}$-operator, we shall show that $\gamma_{\infty}(T) \geqslant B(T)$. To this end we choose an $\varepsilon$ with $0<\varepsilon<B(T)$. By the definition of $B(T)$ there exists a closed subspace $W$ of finite codimension in $X$ such that

$$
B(T) \geqslant m\left(T J_{W}\right)>B(T)-\varepsilon>0 .
$$

In particular, this implies that $N(T) \cap W=\{0\}$. Let $V$ be a finite-dimensional subspace such that

$$
X=W \oplus V \oplus N(T) .
$$

Given $x=w+z$ with $w$ in $W$ and $z$ in $V \oplus N(T)$ we define

$$
F x=-T z \text {. }
$$

Then $F$ is a finite rank operator. Moreover, $N(T+F)=V \oplus N(T)$. By the definition of the reduced minimum modulus we have

$$
\gamma(T+F)=\inf \{\|(T+F) x\|: \operatorname{dist}(x, N(T+F))=1\} .
$$

Every $x$ on the right-hand side can be written as $x=w+z$ with $w$ in $W$ and $z$ in $V \oplus N(T)=N(T+F)$. Therefore $\|w\|=\|x-z\| \geqslant 1$, and hence

$$
\|(T+F) x\|=\|T w\| \geqslant m\left(T J_{w}\right)>B(T)-\varepsilon .
$$

This proves that $\gamma(T+F) \geqslant B(T)-\varepsilon$. Since $\varepsilon$ can be arbitrarily small, we conclude that $\gamma_{\infty}(T) \geqslant B(T)$.

It remains to show that $\gamma_{\infty}(T) \geqslant M(T)$ for any $\Phi_{-}$-operator $T$. The argument follows the preceding one. Choosing $0<\varepsilon<M(T)$, there is, by the definition of $M(T)$, a finite-dimensional subspace $V$ in $X$ such that

$$
M(T) \geqslant q\left(Q_{V} T\right)>M(T)-\varepsilon>0 .
$$

In particular, the operator $Q_{V} T$ is surjective, and hence $X=V+R(T)$. We find a finite-dimensional subspace $W$ in $X$ such that

$$
T^{-1}(V)=W \oplus N(T)
$$

and write

$$
X=W \oplus Z,
$$

where $Z$ is a closed subspace containing $N(T)$. For every $x=w+z$ with $w$ in 
$W$ and $z$ in $Z$ we define

$$
F x=-T w .
$$

Then $F$ is a finite rank operator and

$$
T^{-1}(V)=N(T+F)
$$

By the definition of $\gamma(T+F)$ there exists an $x$ in $X$ such that dist $(x, N(T+F))$ $=1$ and

$$
\gamma(T+F)+\varepsilon>\|(T+F) x\| \geqslant \gamma(T+F)
$$

Writing $x=w+z$ with $w$ in $W$ and $z$ in $Z$, we obtain $(T+F) x=T z$ and $\|z\|=\|x-w\| \geqslant 1$, because $w$ is in $N(T+F)$. Moreover, for every $y$ in $N\left(Q_{V} T\right)=T^{-1}(V)=N(T+F)$ we have $w+y$ in $N(T+F)$, and hence $\|x-(w+y)\| \geqslant 1$, that is, $\|z-y\| \geqslant 1$. So we have dist $\left(z, N\left(Q_{V} T\right)\right) \geqslant 1$ from which we conclude that

$$
\gamma(T+F)+\varepsilon>\|T z\| \geqslant q\left(Q_{V} T\right)>M(T)-\varepsilon .
$$

Since $\varepsilon$ can be arbitrarily small, we obtain $\gamma_{\infty}(T) \geqslant M(T)$. The proof is complete.

The preceding theorem is not indispensable to proving the asymptotic formulas. We have included it here, because it provides an interesting comparison of the reduced minimum modulus to the other semi-Fredholm characteristics. However, the following simple lemma will be crucial in Section 8 .

6.2. Lemma. Let $T$ be a $\Phi_{-}$-operator with $\operatorname{codim} R(T)>0$. Then

$$
M(T) \geqslant(\operatorname{codim} R(T))^{-1} \gamma(T) .
$$

If $R(T)=X$ then $M(T) \geqslant \gamma(T)$, by the definition of $M(T)$.

Proof. Let $\operatorname{codim} R(T)=n$ and $\varepsilon>0$. By [9], Lemma B.4.10, there exists a projection $P$ of the space $X$ onto $R(T)$ such that

$$
\|P\| \leqslant(1+\varepsilon) n .
$$

Let $V=(I-P) X$. Then $X=V \oplus R(T)$ and

$$
N\left(Q_{V} T\right)=N(T) \text {. }
$$

For every $y$ in $R(T)$ and $v$ in $V$ we have $y=P(y-v)$; hence

$$
\|y\| \leqslant\|P\| \cdot\|y-v\| \text {. }
$$

Now, let $x$ in $X$ be such that $\operatorname{dist}(x, N(T))=1$. Denoting $y=T x$, we have by $(8)$

$$
\gamma(T) \leqslant\|T x\| \leqslant\|P\| \cdot \operatorname{dist}(T x, V)
$$

By virtue of (7) this implies

$$
\gamma(T) \leqslant\|P\| q\left(Q_{V} T\right) \leqslant\|P\| \cdot M(T) .
$$

Thus we arrive at

$$
M(T) \geqslant((1+\varepsilon) n)^{-1} \gamma(T) .
$$

Since $:>0$ was arbitrary, the lemma follows.

Remark. If $X$ is a Hilbert space, then the projection $P$ in the preceding proof exists with $\|P\|=1$. So in this situation we get, in fact, $M(T) \geqslant \gamma(T)$. Consequently, by Theorem 3.1 (iv) and Theorem 6.1 , we obtain $M(T)$ $=\gamma_{\infty}(T)$ for any $\Phi_{-}$-operator on a Hilbert space. (Similarly, $B(T)=\gamma_{\infty}(T)$ for $\Phi_{+}$-operators, ef. Corollary 6.3 below.) Moreover, by [15] and [3], Proposition 6.10(i), we have $\gamma_{\infty}(T)=d(T)$ for any semi-Fredholm operator $T$ on a Hilbert space. So in this case the asymptotic formulas for the semiFredholm radius can be derived in an easier way from [16].

6.3. Corollary. Let $T$ be a $\Phi_{+}$-operator with $\operatorname{dim} N(T)>0$. Then

$$
B(T) \geqslant(\operatorname{dim} N(T))^{-1} \gamma(T) \text {. }
$$

If $N(T)=\{0\}$, then $B(T) \geqslant \gamma(T)$, by the definition of $B(T)$.

Proof. This is a consequence of Lemma 6.2, Theorem 4.2(iii), [1], Proposition 1.2.7, and the Kato property $\gamma(T)=\gamma\left(T^{\prime}\right)$.

\section{Removing jumping points}

For every $T$ in $B(X)$, let $r(T)$ denote the surjectivity radius, and $b(T)$ the injectivity radius of the operator $T$. Thus $r(T)$ is the supremum of all $\varepsilon \geqslant 0$ such that $q(T-\lambda I)>0$ for $|\lambda|<\varepsilon$, and $b(T)$ is the supremum of all $\varepsilon \geqslant 0$ such that $m(T-\lambda I)>0$ for $|\lambda|<\varepsilon$. If the operator $T$ is not surjective or bounded from below we define $r(T)=0$ or $b(T)=0$, respectively.

7.1. THEOREM. Let $T$ be a surjective operator. Then

$$
s(T)=\sup _{F} r(T+F),
$$

where the supremum is taken over all finite rank operators.

Proof. Let $D$ denote the open disk of radius $s(T)$ centred at zero. For every $\lambda$ in $D$ we know that $T-\lambda I$ is a $\Phi_{-}$-operator. If $R(T-\lambda I)=X$ we call $\lambda$ a surjectivity point of the operator $T$, while if $\operatorname{codim} R(T-\lambda I)>0$ we say that $\lambda$ is a jumping point of $T$. Of course, we have $r(T) \leqslant s(T)$.

If there are no jumping points in $D$ we have $s(T)=r(T)$ and there is nothing to prove (we note that $s(T+F)=s(T)$ for any finite rank operator $F$ ).

In general, it is well known that the set of jumping points has no limit 
point in $D$, cf. [4], Theorems 3 and 5. So we can label these points in such a way that

$$
\left|\lambda_{1}\right| \leqslant\left|\lambda_{2}\right| \leqslant \ldots<s(T),
$$

and in this notation we have $r(T)=\left|\lambda_{1}\right|$.

We shall show a construction of a finite rank operator $F$ such that

$$
R(T+F-\lambda I)=X
$$

holds for $\lambda=\lambda_{1}$, and also for all $\lambda$ in $D$ for which we already had $R(T-\lambda I)$ $=X$. In other words, the finite rank perturbation $F$ removes the first jumping point and retains all the surjectivity points of the operator $T$ in $D$. Applying such a construction finitely many times, we see that $r(T+F)$ can be made arbitrarily close to $s(T)$ which will prove the theorem.

The construction is based on a reduction theorem of $\mathrm{T}$. Kato [4], Theorem 4. We shall apply it to the operator $T-\lambda_{1} I$. By that theorem the space $X$ decomposes into the direct sum of two closed subspaces $X_{0}$ and $X_{1}$ which are $\left(T-\lambda_{1} I\right)$-invariant, hence $T$-invariant, and have the following properties (we quote only those relevant to our problem). The space $X_{1}$ is finite-dimensional (and $T-\lambda_{1} I$ is nilpotent on it). If $T_{0}$ is the restriction of $T$ to $X_{0}$ considered as an operator from $X_{0}$ into itself, then

$$
\text { codim } R\left(T_{0}-\lambda I\right) \text { is constant in a neighbourhood of } \lambda_{1}
$$

(codimension with respect to $X_{0}$ ).

We note that for any $\lambda$ which is a surjectivity point of $T$ we have necessarily both $(T-\lambda I) X_{1}=X_{1}$ and $(T-\lambda I) X_{0}=X_{0}$. The latter property is of importance. In particular, it implies that $T_{0}-\lambda I$ is surjective on $X_{0}$ for $\lambda$ in a deleted neighbourhood of $\lambda_{1}$. So by (9) we get

$$
\left(T_{0}-\lambda_{1} I\right) X_{0}=X_{0} \text {. }
$$

Now, let $\mu_{1}$ be any complex number with $\left|\mu_{1}\right|>\|T\|+s(T)$. We define the finite rank operator $F$ to be zero on $X_{0}$, and $\mu_{1} I$ on $X_{1}$. Then, for every $\lambda$ with $|\lambda|<s(T)$ we have

$$
(T+F-\lambda I) X_{1}=X_{1}
$$

because the spectrum of the restriction of $T-\lambda I$ to the finite-dimensional subspace $X_{1}$ is contained in the disk of radius $\|T-\lambda I\|<\|T\|+s(T)<\left|\mu_{1}\right|$ so that the operator $T+F-\lambda I$ is certainly invertible on $X_{1}$.

Since $F$ is zero on $X_{0}$, we also have

$$
(T+F-\lambda I) X_{0}=X_{0}
$$

for every surjectivity point $\lambda$ of the operator $T$, and also for $\lambda=\lambda_{1}$ by (10). So the operator $T+F$ is as desired.

Similarly one can prove the following
7.2. ThEOREM. Let $T$ be an operator bounded from below. Then

$$
s(T)=\sup _{F} b(T+F)
$$

where the supremum is taken over all finite rank operators.

\section{The asymptotic formulas}

In this section we prove all the formulas promised in Sections 1 and 2 .

8.1. Theorem. For every $T$ in $B(X)$ we have

and

$$
\begin{aligned}
& \lim _{n} M\left(T^{n}\right)^{1 / n}=\lim _{n} K\left(T^{n}\right)^{1 / n}=s_{-}(T), \\
& \lim _{n} B\left(T^{n}\right)^{1 / n}=\lim _{n} G\left(T^{n}\right)^{1 / n}=s_{+}(T) .
\end{aligned}
$$

Proof. If $T$ is not semi-Fredholm, then everything is zero. Let us therefore suppose that $T$ is a $\Phi_{-}$-operator and prove (11) with $s_{-}(T)=s(T)$, of course. By (6), Theorem 5.3, and (1) we have

$$
M(T) \leqslant K(T) \leqslant d(T) \leqslant s(T) .
$$

We shall apply these inequalities to the powers of $T$ but before we note that $s\left(T^{n}\right)=s(T)^{n}$ for $n=1,2, \ldots$ This can easily be checked by using the factorization

$$
T^{n}-\lambda^{n}=(T-\lambda)\left(T^{n-1}+\ldots+\lambda^{n-1}\right)
$$

and the division property of $\Phi_{-}$-operators [1], Corollary 1.3.5. (Another verification reducing the problem to the analogous property of the surjectivity radius [6] can be found in [16].) So it follows that

$$
\overline{\lim } M\left(T^{n}\right)^{1 / n} \leqslant s(T)
$$

and it remains to show that

$$
\underline{\lim } M\left(T^{n}\right)^{1 / n} \geqslant s(T) .
$$

Suppose first that ind $T<0$ which is the more sophisticated case. By [13], Theorem 3.9 , there is a finite rank operator $V$ such that the operator $S=T+V$ is bounded from below. To this operator $S$ we apply Theorem 7.2. Given $\varepsilon>0$, there exists a finite rank operator $F$ such that

$$
s(S) \geqslant b(S+F)>s(S)-\varepsilon .
$$

Let $A=S+F$. Since $s(S)=s(T)$, we can write

$$
s(T) \geqslant b(A)>s(T)-\varepsilon .
$$


For the injectivity radius $b(A)$ we have the formula

$$
b(A)=\lim _{n} \gamma\left(A^{n}\right)^{1 / n}
$$

from [6], Theorem 3. By Lemma 6.2 of this paper we have

$$
M\left(A^{n}\right) \geqslant\left(\operatorname{codim} R\left(A^{n}\right)\right)^{-1} \gamma\left(A^{n}\right) .
$$

Intuitively, $\operatorname{codim} R\left(A^{n}\right)$, being always finite, cannot increase too quickly as $n$ tends to infinity. More precisely, by [12], Lemma 3.3, we have

$$
\operatorname{codim} R\left(T^{n}\right) \leqslant n \cdot \operatorname{codim} R(T)
$$

from which we conclude that

$$
\lim _{n}\left(\operatorname{codim} R\left(A^{n}\right)\right)^{1 / n}=1 .
$$

Noting that $M\left(A^{n}\right)=M\left(T^{n}\right)$ by Theorem 3.1(iv), we arrive at

$$
\underline{\lim } M\left(T^{n}\right)^{1 / n} \geqslant b(A)>s(T)-\varepsilon .
$$

Since $\varepsilon>0$ was arbitrary, this implies (13) in the case when ind $T<0$.

If ind $T \geqslant 0$, then by [13], Theorem 3.10 , there exists a finite rank operator $V$ such that the operator $T+V$ is surjective. Now we apply the same argument as before based on Theorem 7.1, the trivial part of Lemma 6.2 , and [6], Theorem 1. Thus we have proved (11).

Formulas (12) follow now from (11) applied to the adjoint operator and by using Theorem 4.2(iii). have

8.2. TheOREM. For every semi-Fredholm operator $T$ on a Banach space we

$$
\lim _{n} \gamma_{\infty}\left(T^{n}\right)^{1 / n}=\lim _{n} d\left(T^{n}\right)^{1 / n}=s(T)
$$

Proof. This is a consequence, of Theorems 6.1 and 8.1. Another proof follows directly from the theorems of Section 7 and the formulas obtained in [6], cf. Theorem 8.3 below.

Let $T$ be a bounded linear operator on a Banach space: We know that $T$ is semi-Fredholm if and only if there is a finite rank operator $F$ such that the operator $T+F$ is either surjective or bounded from below. More precisely, writing

$$
q_{\infty}(T)=\sup _{F} q(T+F) \quad \text { and } \quad m_{\infty}(T)=\sup _{F} m(T+F),
$$

where $F$ varies over the finite rank operators, we have $q_{\infty}(T)>0$
$\left(m_{\infty}(T)>0\right)$ if and only if ind $T \geqslant 0$ (ind $T \leqslant 0$ ). Let .

$$
g(T)=\max \left\{q_{\infty}(T), m_{\infty}(T)\right\} .
$$

Clearly, we have $g(T) \leqslant \gamma_{\infty}(T)$, and $g(T)>0$ if and only if $T$ is semiFredholm. The method of removing jumping points (Section 7) combined with the formulas from [6] still gives the following

8.3. Theorem. For every $T$ in $B(X)$ we have

$$
\lim _{n} g\left(T^{n}\right)^{1 / n}=s(T)
$$

Finally we note that the existence of the limit in Theorem 8.3 has been observed by V. Rakočević [10] though without determining its meaning.

\section{References}

[1] S. R. Caradus, W. E. Pfaffenberger and B. Yood, Calkin algebras and algebras of operators on Banach spaces, M. Dekker, New York 1974.

[2] S. Goldberg, Unbounded linear operators, McGraw-Hill, New York 1966.

[3] D. A. Herrero, Approximation of Hilbert space operators I, Pitman, London 1982.

[4] T. Kat o, Perturbation theory for nullity, deficiency and other quantities of linear operators, J. Analyse Math. 6 (1958), 261-322.

[5] H. König, A formula for the eigenvalues of a compact operator, Studia Math. 65(1979), $141-146$.

[6] E. Makai, jr. and J. Zemánek, The surjectivity radius, packing numbers and boundedness below of linear operators, Integral Equations Operator Theory 6 (1983), 372-384.

[7] A. Pełczyński, On strictly singular and strictly cosingular operators. II. Strictly singular and strictly cosingular operators in L(v)-spaces, Bull. Acad. Polon. Sci. Sér. Sci. Math. Astronom. Phys. 13 (1965), 37-41.

[8] A. Pietsch, s-Numbers of operators in Banach spaces, Studia Math. 51 (1974), 201-223. [9] -, Operator ideals, VEB Deutscher Verlag der Wissenschaften, Berlin 1978.

[10] V. Rakočević, On one subset of M. Schechter's essential spectrum, Mat. Vesnik 5 (1981) 389-391.

[11] M. Schechter, Quantities related to strictly singular operators, Indiana Univ. Math. J. 21 (1972), 1061-1071.

[12] A. E. Taylor, Theorems on ascent, descent, nullity and defect of linear operators, Math Ann. 163 (1966), 18-49.

[13] B. Yood, Properties of linear transformations preserved under addition of a completely continuous transformation, Duke Math. J. 18 (1951), 599-612.

[14] J. Zemánek. The essential spectral radius and the Riesz part of the spectrum, in: Functions, Series, Operators (Proc. Internat. Conf., Budapest 1980), Colloq. Math. Soc. János Bolyai, vol. 35, North-Holland, Amsterdam 1983, 1275-1289.

[15] -, Geometric interpretation of the essential minimum modulus, in: Invariant Subspaces and Other Topics (Timisoara/Herculane, 1981), Operator Theory: Adv. Appl, vol 6, Birkhäuser, Basel 1982, 225-227.

[16] -, The semi-Fredholm radius of a linear operator, Bull. Pol.* Acad. Sci. Math 32 (1984), 67-76. 
[17] J. Ze mánek, The stability radius of a semi-Fredholm operator, Integral Equations Operator Theory, to appear.

[18] A. S. Fainstein, On measures of noncompactness of linear operators and analogues of the minimum modulus for semi-Fredholm operators (in Russian), in: Spektral'naya Teoriya Operatorov i Prilozh., vol. 6, izdat. Elm, Baku, to appear.

INSTYTUT MATEMATYCZNY POLSKIEJ AKADEMII NAUK

INSTITUTE OF MATHEMATICS

POLISH ACADEMY OF SCIENCES

00-950 Warszuwa, P. O. Box 137

Poland

\section{Nilpotent Lie groups and summability of eigenfunction} expansions of Schrödinger operators*

by

ANDRZEJ HULANICKI (Wrocław) and JOE W. JENKINS (Albany, N. Y.)

Abstract. Let $A$ be an operator densely defined on $L^{2}(X)$, where $X$ is a measure space, which is essentially self-adjoint on its domain and non-negative. Let

$$
A f=\int_{0}^{\infty} \lambda d E(\lambda) f
$$

be the spectral expansion of $A$. We study conditions on functions $K$ on $\boldsymbol{R}^{+}$such that

$$
\lim _{t \rightarrow 0} \int_{0}^{\infty} K(t \lambda) d E(\lambda) f=f \quad \text { a.e. }
$$

for functions $f \in L^{p}(X), 1 \leqslant p<\infty$, for operators $A$ which are of the form $\pi(L)$, where

$$
L=\sum_{j=1}^{k}(-1)^{n_{j}} X_{j}^{2 n_{j}}
$$

$X_{1}, \ldots, X_{k}$ are generators of the Lie algebra of a nilpotent Lie group $G$ and $\pi$ is a representation of $G$ induced by a unitary character of a normal connected subgroup of $G$. As a corollary we obtain the following. Let

$$
A=\sum_{j=0}^{k}(-1)^{n_{j}} \partial^{2 n_{j}} / \partial x_{j}^{2 n_{j}}+V
$$

where the potential $V$ is a sum of squares of real polynomials on $R^{k}$. Then there exists an $N$ such that

$$
\lim _{t \rightarrow 0} \int_{0}^{\infty}(1-\lambda)_{+}^{N} d E(\lambda) f=f \quad \text { a.e., } \quad f \in L^{1}\left(\boldsymbol{R}^{k}\right),
$$

where $E$ is the spectral measure of $A$ on $L^{2}\left(R^{k}\right)$.

Let $A$ be an operator densely defined on $L^{2}(X)$, where $X$ is a measure space, which is essentially self-adjoint on its domain and non-negative. Let

$$
A f=\int_{0}^{\infty} \lambda d E(\lambda) f
$$

be the spectral expansion of $A$.

\footnotetext{
* This research was funded in part by National Sciences Foundation Grant No. 810078.
} 